\title{
THE COSMOLOGICAL CONSTANT AND STABILITY OF THE HIGGS VACUUM *
}

\author{
Steven D. BAss \\ Stefan Meyer Institute for Subatomic Physics, \\ Austrian Academy of Sciences, Boltzmanngasse 3, 1090 Vienna, Austria
}

\begin{abstract}
We discuss the cosmological constant puzzle and possible connections to the (meta-)stability of the Higgs vacuum suggested by recent LHC results. A possible explanation involves new critical phenomena in the ultraviolet, close to the Planck scale.
\end{abstract}

PACS numbers: 11.15.Ex, 14.80.Bn, 95.36.+x, 98.80.Es

\section{Introduction}

The cosmological constant puzzle connects particle physics and cosmology. The accelerating expansion of the Universe is interpreted within Einstein's theory of General Relativity as driven by a small positive cosmological constant or vacuum energy density perceived by gravitational interactions called dark energy, for reviews see Refs. [1, 2]. Understanding this vacuum energy is an important challenge for theory and connects the Universe on cosmological scales (the very large) with quantum field theory and subatomic physics (the very small).

Current observations point to an energy budget of the Universe where just $5 \%$ is composed of matter built from quarks and leptons, $26 \%$ involves dark matter (possibly made of new elementary particles) and $69 \%$ is dark energy [3]. The vacuum energy density extracted from astrophysics is

$$
\rho_{\mathrm{vac}}=\mu_{\mathrm{vac}}^{4} \sim(0.002 \mathrm{eV})^{4} .
$$

The scale $\mu_{\mathrm{vac}}$ is similar to the value we expect for the light neutrino mass whereas $\rho_{\mathrm{vac}}$ is $10^{56}$ times smaller than the value expected from the classical Higgs potential of Standard Model particle physics which also comes with

\footnotetext{
* Presented at the Jagiellonian Symposium on Fundamental and Applied Subatomic
} Physics, Cracow, June 7-12 2015. 
the opposite negative sign. What might dark energy be telling us about the intersection of particle physics and gravitation?

General Relativity and the particle physics Standard Model work excellently everywhere they have been tested in experiments. The Higgs boson discovered at the LHC [4] is consistent with Standard Model expectations [5. It is an open question whether at a deeper level this boson is elementary or of dynamical origin. Results from the LHC experiments ATLAS, $\mathrm{CMS}$ and $\mathrm{LHCb}$ are in good agreement with the Standard Model with (so far) no evidence of new physics. Precision measurements of the electron electric dipole moment are consistent with zero and constrain possible new sources of CP violation from beyond the Standard Model up to scales similar to or larger than those probed at the LHC [6]. Whilst the Standard Model has proved very successful everywhere it has been tested we know that some extra physics is needed to explain the very small neutrino masses, the baryon asymmetry and strong $\mathrm{CP}$ problems as well as dark matter and inflation. The scale of this new physics is as yet unknown and not yet given by experiments.

\section{The cosmological constant and particle physics}

Interestingly, the dark energy scale $\mu_{\mathrm{vac}} \sim 0.002 \mathrm{eV}$ is similar to the value that we expect for the light neutrino mass [7-9] taking the normal hierarchy of neutrino masses [10. One observes the phenomenological relation [2, 8]

$$
\mu_{\mathrm{vac}} \sim m_{\nu} \sim \Lambda_{\mathrm{ew}}^{2} / M,
$$

where $\Lambda_{\text {ew }}$ is the electroweak scale and $M \sim 3 \times 10^{16} \mathrm{GeV}$ is logarithmically close to the Planck mass $M_{\mathrm{Pl}} \sim 1.2 \times 10^{19} \mathrm{GeV}$ and typical of the scale that appears in Grand Unified Theories and in the see-saw mechanism for neutrino mass generation [11. If taken literally Eq.(2) connects neutrino physics, Higgs phenomena in electroweak symmetry breaking and dark energy to a new high mass scale which needs to be understood. The gauge bosons in the Standard Model which have a mass through the Higgs mechanism are also the gauge bosons which couple to the neutrino.

The formula Eq.(2) was also suggested by Bjorken [12] without connection to neutrinos in the "gaugeless limit" of the Standard Model with composite or emergent gauge bosons being born at a large mass scale $M$ and no or only very small coupling to new physics between the electroweak and ultraviolet mass scales.

There are theoretical hints that the large mass scale in Eq.(2) might perhaps be associated with dynamical symmetry breaking, see below. The see-saw formula Eq.(2), if evidence of deeper physics, suggests that the cosmological constant puzzle and the electroweak hierarchy problem might 
be connected and perhaps be resolved at a scale close to the Planck scale rather than around the $\mathrm{TeV}$ scale.

Here one finds a possible connection with LHC results. With the values of the top-quark and Higgs boson masses measured at the LHC, the Standard Model works as a consistent perturbative theory up to very high scales. Renormalisation group (RG) calculations reveal that the Standard Model Higgs vacuum with no coupling to new interactions sits close to the border of being stable and metastable (with half-life much greater than the present age of the Universe) [13-20]. An unstable vacuum would require coupling to some new interaction above the electroweak scale. Vacuum stability is very sensitive to the exact values of the Higgs and top-quark masses and technical details in calculating $\overline{\mathrm{MS}}$ parameters in terms of physical ones and how one should include tadpole diagrams to be consistent with gauge invariance. The key issue is that the $\beta$ function for the Higgs four-boson self-coupling $\lambda$ has a zero around $10^{17} \mathrm{GeV}$, close to the Planck mass, and when (if at all) this coupling $\lambda$ crosses zero, perhaps around $10^{10}-10^{12} \mathrm{GeV}$ [13] or, for a stable vacuum, not at all [16, 18]. With modest changes in the top-quark and Higgs masses (increased top mass and/or reduced Higgs mass) the Standard Model vacuum would become unstable. In a recent calculation Bednyakov et al. 20] find the value of the top quark mass for the vacuum to be stable all the way up to the Planck mass to be within $1.3 \sigma$ of the Monte Carlo mass quoted by the Particle Data Group. With the vacuum either stable up to the Planck scale or at the border of stable and metastable, then some critical process might be at work in the ultraviolet [13, 18.

Ideas connecting the cosmological constant and Higgs-mass hierarchy problem to new critical phenomena near the Planck scale are discussed in Refs. [2, 8, 12, 18, 21]. Perhaps the Standard Model is itself (in part) emergent as the long range tail of a critical system that exists close to the Planck scale and there is no new scale between the electroweak scale and some very high scale close to the Planck mass?

In parallel to RG discussions of vacuum (meta-)stability, the RG behaviour of the perturbative coefficient of the quadratically divergent counterterm for the Higgs mass squared is also interesting. This coefficient crossing zero [22] in the ultraviolet would trigger a first order phase transition restoring electroweak symmetry [18]. Whether this crossing transition happens above or below the Planck scale depends strongly on the value of the top-quark mass and matching between the $\overline{\mathrm{MS}}$ and physical parameters [13, 17, 18, 23]. In the calculation of Jegerlehner [18] with a stable vacuum the crossing transition takes place around $10^{16} \mathrm{GeV}$, close to the mass scale $M$ in Eq.(2).

We next consider how Eq.(2) might be understood, treating the chiral- 
ity of the neutrino by analogy as an Ising-like "spin" degree of freedom that becomes active near the Planck scale [8]. Analogies between quantum field theories and condensed matter and statistical systems have often played an important role in motivating ideas in particle physics. The ground state of the Ising model exhibits spontaneous magnetisation where all the spins line up and the internal energy per spin and the free energy density of the spin system go to zero with corrections dampened by a strong suppression exponential factor, viz. $e^{-\beta J}$ where $\beta$ is Boltzmann's constant and $J$ is the scale of the Ising interaction. In mappings between statistical mechanics and quantum field theory the free energy density for the statistical "spin" system plays the role of the vacuum energy density in quantum field theory 24] suggesting possible application to the cosmological constant puzzle. For an Ising system with no external magnetic field the free energy density is equal to minus the pressure. The model equation of state looks like a vacuum energy term in Einstein's equations of General Relativity, proportional to the metric tensor $g_{\mu \nu}$. Taking the scale of the Ising interaction $J \sim M$ close to the Planck mass and coupling the "neutrino" spins to gauge fields it is plausible that the gauge bosons which couple to the "neutrino" nonperturbatively acquire mass in the ground state [2, 8]. That is, they are in a Higgs phase. Further, the lowest energy eigenvalue characterising the free energy of the combined "spin"-gauge system then looks like the see-saw formula, Eq.(2), with large mass scale $M \sim 10^{16} \mathrm{GeV}$.

If the gauge symmetries of the Standard Model are emergent, this differs from the paradigm of unification with maximum symmetry at the highest possible energies with a unification big gauge group spontaneously broken through various Higgs condensates to the Standard Model, with each new condensate introducing an extra large contribution to the vacuum energy and the cosmological constant. With emergent gauge fields [18, 25-28] Lorentz invariance is expected to be (spontaneously) broken or emergent close to the critical mass scale in the ultraviolet [26]. Bjorken has argued that any violations of Lorentz and gauge symmetries in the emergence scenario might appear with coefficient suppressed by powers of the cosmological constant scale divided by the large scale $M$ close to the Planck mass [26, 27], thus vanishing in the limit of vanishing dark energy. For emergent QED a preferred reference frame is naturally identified as the frame for which the cosmic microwave background is locally at rest. Non-renormalisable contributions from high dimensional operators would be proportional to powers of energy divided by $M$ and are very much suppressed much below the Planck scale [18]. In the emergence scenario fundamental symmetries like gauge and Lorentz invariance would "dissolve" in the ultraviolet and could be manifest as infrared attractive fixed points of the renormalisation group behaviour of a larger class of possible theories [28]. Perhaps the gauge the- 
ories of particle physics and also General Relativity are effective theories with characteristic energy of order the Planck scale [29].

\section{Conclusions}

The cosmological constant puzzle continues to challenge our understanding of fundamental physics. Why is the dark energy density finite, positive and so very small? Might the value of the cosmological constant and electroweak symmetry breaking be related, perhaps with common origin connected to new critical phenomena in the ultraviolet close to the Planck scale? The cosmological constant puzzle promises to teach us a great deal about the intersection of particle physics and gravitation.

\section{Acknowledgements}

I thank Pawel Moskal for the invitation to this stimulating meeting in the beautiful surroundings of the Collegium Maius.

\section{REFERENCES}

[1] S. Weinberg, Rev. Mod. Phys. 61, 1 (1989);

V. Sahni and A. Starobinsky, Int. J. Mod. Phys. D9, 373 (2000);

S. M. Carroll, Living Rev. Relativity 4, 1 (2001);

P. J. E. Peebles and B. Ratra, Rev. Mod. Phys. 75, 559 (2003);

E. J. Copeland, M. Sami and S. Tsujikawa, Int. J. Mod. Phys. D15, 1753 (2006);

N. Straumann, Lect. Notes Phys. 721, 327 (2007);

J. A. Frieman, M. S. Turner and D. Huterer, Ann. Rev. Astron. Astrophys. 46, 385 (2008);

R. Bean, arXiv: 1003.4468 [astro-ph.CO];

S. D. Bass, J. Phys. G 38, 043201 (2010);

J. Lesgourgues, PoS EPS -HEP2011, 015 (2011);

J. Martin, Comptes Rendus Physique 13, 566 (2012);

J. Sola, J. Phys. Conf. Ser. 453, 012015 (2013).

[2] S. D. Bass, Mod. Phys. Lett. A30, 1540033 (2015).

[3] Planck Collab. (P. A. R. Ade et al.), arXiv:1502.01589 [astro-ph.CO].

[4] ATLAS Collab. (G. Aad et al.), Phys. Lett. B 716, 1 (2012);

CMS Collab. (S. Chatrchyan et al.), Phys. Lett. B 716, 30 (2012).

[5] G. Altarelli, Physica Scripta T158, 014011 (2013); arXiv:1407.2122 [hep-ph].

[6] ACME Collab. (J. Baron et al.), Science 343, 6168 (2014).

[7] G. Altarelli, Nucl. Phys. B (Proc. Suppl.) 143, 470 (2005).

[8] S. D. Bass, arXiv:1210.3297 [hep-ph], Acta Phys. Pol. B 45, 1269 (2014). 
[9] D. G. Caldi and A. Chodos, hep-ph/9903416

R. Fardon, A. E. Nelson and N. Weiner, JCAP 10, 005 (2004);

C. Wetterich, Phys. Lett. B655, 201 (2007).

[10] G. Altarelli, arXiv:1111.6421 [hep-ph];

H. Fritzsch and S. Zhou, Phys. Lett. B718, 1457 (2013).

[11] P. Minkowski, Phys. Lett. B67, 421 (1977);

M. Gell-Mann, P. Ramond and R. Slansky, in Supergravity, edited by D. Freedman and P. van Nieuwenhuizen (North Holland, Amsterdam, 1979), p. 315; T. Yanagida, in Proceedings of the Workshop on Unified Theory and Baryon Number in the Universe, edited by O. Sawada and A. Sugamoto (KEK, Tsukuba, Japan, 1979);

R. Mohapatra and G. Senjanovic, Phys. Rev. Lett. 44, 912 (1980).

[12] J. D. Bjorken, Phys. Rev. D64, 085008 (2001).

[13] G. Degrassi et al., JHEP 08, 098 (2012);

D. Buttazzo et al., JHEP 12, 089 (2013).

[14] F. Bezrukov, M. Yu. Kalmykov, B. A. Kniel and M. Shaposhnikov, JHEP 10, 140 (2012);

F. Bezrukov and M. Shaposhnikov, J. Exp. Theor. Phys. 120, 335 (2015).

[15] S. Alekhin, A. Djouadi and S. Moch, Phys. Lett. B716, 214 (2012).

[16] I. Masina, Phys. Rev. D87, 053001 (2013).

[17] Y. Hamada, H. Kawai and K-j. Oda, Phys. Rev. D87, 053009 (2013); (E) Phys. Rev. D89, 059901 (2014).

[18] F. Jegerlehner, Acta Phys. Pol. B 45, 1167 (2014).

[19] F. Jegerlehner, M. Kalmykov and B. A. Kniehl, J. Phys. Conf. Series 608, $012074(2015)$.

[20] A. V. Bednyakov, B. A. Kniehl, A. F. Pikelner and O. L. Veretin, Phys. Rev. Lett. 115, 201802 (2015).

[21] F. Jegerlehner, Acta Phys. Pol. B 45, 1215 (2014).

[22] M. J. G. Veltman, Acta Polon. B 12, 437 (1981).

[23] I. Masina and M. Quiros, Phys. Rev. D88, 093003 (2013).

[24] J. B. Kogut, Rev. Mod. Phys. 51, 659 (1979).

[25] F. Jegerlehner, hep-th/9803021:

F. Jegerlehner, Helv. Phys. Acta 51, 783 (1978).

[26] J. Bjorken, hep-th/0111196.

[27] J. D. Bjorken, arXiv:1008.0033 [hep-ph];

J. D. Bjorken, The Future and its Alternatives in http://www.slac.stanford.edu/grp/th/symposia/QuinnSymposium.htm

[28] S. Chadha and H. B. Nielsen, Nucl. Phys. B217, 125 (1983);

J. L. Chkareuli, C. D. Frogatt and H. B. Nielsen, Phys. Rev. Lett. 87, 091601 (2001).

[29] S. Weinberg, arXiv:0903.0568 [hep-th]. 